\title{
OPEN A high sensitivity ZENK monoclonal antibody to map neuronal activity in Aves
}

\begin{abstract}
Gregory Charles Nordmann ${ }^{1}$, Erich Pascal Malkemper $\mathbb{1}^{1}$, Lukas Landler ${ }^{1}$, Lyubov Ushakova ${ }^{1}$, Simon Nimpf ${ }^{1}$, Robert Heinen ${ }^{1}$, Stefan Schuechner ${ }^{2}$, Egon Ogris $\mathbb{D}^{2}$ \& David Anthony Keays $\mathbb{D}^{1 *}$

The transcription factor ZENK is an immediate early gene that has been employed as a surrogate marker to map neuronal activity in the brain. It has been used in a wide variety of species, however, commercially available antibodies have limited immunoreactivity in birds. To address this issue we generated a new mouse monoclonal antibody, 7B7-A3, raised against ZENK from the rock pigeon (Columba livia). We show that 7B7-A3 labels clZENK in both immunoblots and histological stainings with high sensitivity and selectivity for its target. Using a sound stimulation paradigm we demonstrate that 7B7-A3 can detect activity-dependent ZENK expression at key stations of the central auditory pathway of the pigeon. Finally, we compare staining efficiency across three avian species and confirm that 7B7-A3 is compatible with immunohistochemical detection of ZENK in the rock pigeon, zebra finch, and domestic chicken. Taken together, 7B7-A3 represents a useful tool for the avian neuroscience community to map functional activity in the brain.
\end{abstract}

Over the past century Aves have served as powerful models to study vertebrate cognitive and sensory neurobiology ${ }^{1}$. It has been shown that crows and cockatoos can develop tools to solve complex tasks ${ }^{2-4}$, pigeons can memorize abstract visual patterns $s^{5,6}$, songbirds are able to learn conspecific and novel vocalizations $s^{7,8}$, and robins rely on magnetic fields for long-range navigation ${ }^{9,10}$. It is apparent from these studies that the avian brain processes information from a myriad of sensory cues allowing it to drive an array of complex behaviours.

The quantitation of immediate early gene (IEG) expression is a well established methodology to map the neuronal networks that integrate this information. IEGs represent a class of genes characterized by a rapid rise in expression levels upon sustained cell stimulation and are therefore linked to synaptic activity in the brain ${ }^{11,12}$. One commonly used IEG is the transcription factor ZENK (an acronym for $z i f-268$, egr-1, ngfi-a, krox-24) ${ }^{13-16}$. Upon expression, this zinc finger protein regulates the transcription of downstream targets, thereby modulating synaptic long-term adaptations to incoming information ${ }^{17}$. ZENK $\mathrm{mRNA}$ is induced rapidly in response to stimulation $^{18}$, followed by peak protein expression approximately $1-2$ hours after stimulus onset ${ }^{19,20}$. It should be noted, however, that not all neuronal populations in the bird brain express ZENK ${ }^{19,21-23}$, and that ZENK induction is dependent on stimulus contex $\mathrm{t}^{24,25}$ or neuromodulatory factors such as the activity of the noradrenergic system ${ }^{26}$.

To date avian studies relying on ZENK expression have employed in situ hybridization, or a commercial polyclonal antibody raised against the rabbit ZENK homologue EGR-1 (C-19) ${ }^{19,27-30}$. While C-19 has been widely used, it displays high batch-to-batch variation, requires laborious amplification methods in some species ${ }^{27,28}$, and its production has now been discontinued by the provider. In order to establish a reliable alternative to C-19 we set out to generate a ZENK antibody specifically for Aves. Here, we describe the generation and validation of a new ZENK monoclonal antibody, 7B7-A3, raised in mice against a ZENK peptide from the rock pigeon (Columba livia). We show that 7B7-A3 labels ZENK in pigeons, zebra finches, and chickens, and it serves as a useful marker to detect auditory-induced neuronal activation.

${ }^{1}$ Research Institute of Molecular Pathology, Vienna Biocenter (VBC), Campus-Vienna-Biocenter 1, 1030, Vienna, Austria. ${ }^{2}$ Monoclonal Antibody Facility, Max Perutz Labs, Medical University of Vienna, Dr. Bohr-Gasse 9, 1030, Vienna, Austria.*email: keays@imp.ac.at 
a

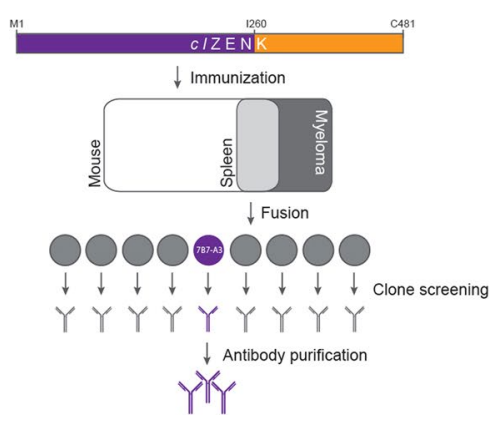

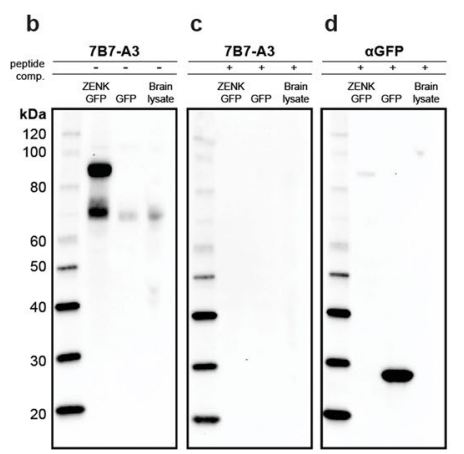

Figure 1. ZENK antibody generation and validation. (a) Diagram depicting the methodology employed to generate the ZENK antibody. A 260 amino acid N-terminal fragment (purple) of the pigeon ZENK protein (orange) was recombinantly expressed and injected into mice. Harvested spleen cells were fused with myeloma cells and hybridomas screened by western blot analysis. This resulted in the identification of clone 7B7-A3 (shown in purple). (b) Western blot analysis of pigeon embryonic fibroblasts expressing ZENK-GFP or GFP alone, and protein lysates from pigeon brain tissue. Blots incubated with 7B7-A3 (left panel) show bands at $\sim 70 \mathrm{kDa}$ and $\sim 95 \mathrm{kDa}$, corresponding to the endogenous and GFP tagged protein, respectively. (c) and (d) Peptide competition experiment. Preincubation of the antibody with the antigen $(+)$ prevents binding of 7B7A3 antibody (c), but not of a GFP antibody (d). (e,f) Permanent immunohistochemical staining using 7B7-A3 leads to labeling of cell nuclei on pigeon brain sections. (g,h) Antigen competition experiment. Preincubation of the antibody with the antigen abolishes $7 \mathrm{~B} 7-\mathrm{A} 3$ staining. $(\mathbf{i}, \mathbf{j})$ No primary antibody control. Scalebars represent $100 \mu \mathrm{m}$ in $(\mathbf{e}),(\mathbf{g})$, and $(\mathbf{i})$; and $50 \mu \mathrm{m}$ in insets $(\mathbf{f}),(\mathbf{h})$, and $(\mathbf{j})$.

\section{Results}

ZENK antibody generation and validation. To determine the full-length protein sequence of ZENK in Columba livia (clZENK) we extracted mRNA from the pigeon brain, generated cDNA, and amplified the transcript using primers designed from existing genomic resources ${ }^{31,32}$. This resulted in the cloning of a 1443 bp gene product that codes for a 481 amino acid protein. An N-terminal fragment 260 amino acids in length (1-260) with high surface probability was chosen as the antigen. The protein was heterologously expressed in E. coli, purified, the sequence verified by mass spectrometry, and then injected into BALB/c mice. The spleens of immunized mice were harvested, hybridomas generated, and clones screened by western blot analysis for sera that bind the clZENK protein (Fig. 1a). This resulted in the identification of clone 7B7-A3. Purified antibodies generated from this clone detect ZENK $(\sim 70 \mathrm{kDa})$, and GFP tagged ZENK $(\sim 95 \mathrm{kDa})$ in protein lysates from pigeon embryonic fibroblasts (PEFs) transiently transfected with clZENK-GFP (Fig. 1b). In lysates generated from the pigeon brain we observed a single $70 \mathrm{kDa}$ band that is consistent with endogenously expressed ZENK. It should be noted that like some commercially available antibodies raised against ZENK the detected size of $70 \mathrm{kDa}$ in western blots exceeds the predicted weight of $57 \mathrm{kDa}$, possibly due to posttranslational modifications. Therefore to validate the selectivity for the $c l Z E N K$ epitope, we performed a peptide competition experiment by preincubating the antibody with the antigen. This preincubation prevents binding of the 7B7-A3 antibody (Fig. 1c), but not binding of a GFP antibody control (Fig. 1d). Next, we assessed the utility of the 7B7-A3 antibody as a marker on histological sections. Employing antigen retrieval and a permanent staining protocol we found that 7B7-A3 antibody reliably labels cell nuclei in pigeon brain sections at dilutions as low as 1:500,000 $(3.2 \mathrm{ng} / \mathrm{ml})$, without the need for post-chromogenic enhancement (Fig. 1e,f). Pre-absorption of the 7B7-A3 antibody with the antigen (Fig. 1g,h), as well as omission of the primary antibody (Fig. 1i,j) resulted in no visible nuclear staining, confirming the selectivity of the 7B7-A3 antibody for the clZENK epitope. Taken together, these data indicate that the 7B7-A3 antibody binds $c l$ ZENK.

Distribution of ZENK in the pigeon brain. Next, we examined the distribution of ZENK expressing neuronal populations in the pigeon brain. We sacrificed animals that were maintained in a standard aviary (an environment with numerous sensory cues) and stained sections from the brainstem, thalamic and forebrain regions involved in higher sensory processing using our 7B7-A3 antibody. Within the brainstem we observed ZENK-positive cells in primary nuclei of the auditory (e.g. nc. magnocellularis, Fig. 2a,b), vestibular (e.g. nc. vestibularis dorsalis, Fig. 2a,c), and trigeminal system (e.g. SpV, Fig. 2a,c). In the midbrain, the 7B7-A3 antibody labels thalamic relays, such as the lateral habenular nucleus (Fig. 2d,e) as well both retinorecipient and nonretinorecipient layers of the rostral optic tectum (Fig. 2d,f). In the forebrain, consistent with previous studies $^{21-23}$, we observed limited immunoreactivity within entopallial structures (Fig. 2g,i). In contrast, we found robust and broad ZENK staining in other areas involved in higher sensory processing such as the rostral piriform corte $^{33}$ and multi-sensory integrating regions of the medial intermedioventral mesopallium ${ }^{34}$, as well as the apical hyperpallium ${ }^{35}$ and the rostral hippocampal formation ${ }^{36}$ (Fig. 2g,h). This broad ZENK expression was markedly reduced after isolating the animals $(n=3)$ for 7 hours in a dark cage in silence. $\mathrm{ZENK}^{+}$cells were reduced by $72 \%$ in the rostral hippocampus $(\mathrm{P}<0.05 ; \mathrm{t}=2.45 ; \mathrm{df}=4$; Supplementary Fig. $1 \mathrm{a}-\mathrm{c})$, by $82 \%$ in the lateral striatum $(\mathrm{P}<0.05 ; \mathrm{t}=3.47 ; \mathrm{df}=4$; Supplementary Fig. $1 \mathrm{~d}-\mathrm{f})$, and by $71 \%$ in the optic tectum $(\mathrm{P}<0.05$; $\mathrm{t}=3.70 ; \mathrm{df}=4$; Supplementary Fig. $1 \mathrm{~g}-\mathrm{i})$. We conclude that ZENK is expressed in various networks of the pigeon 

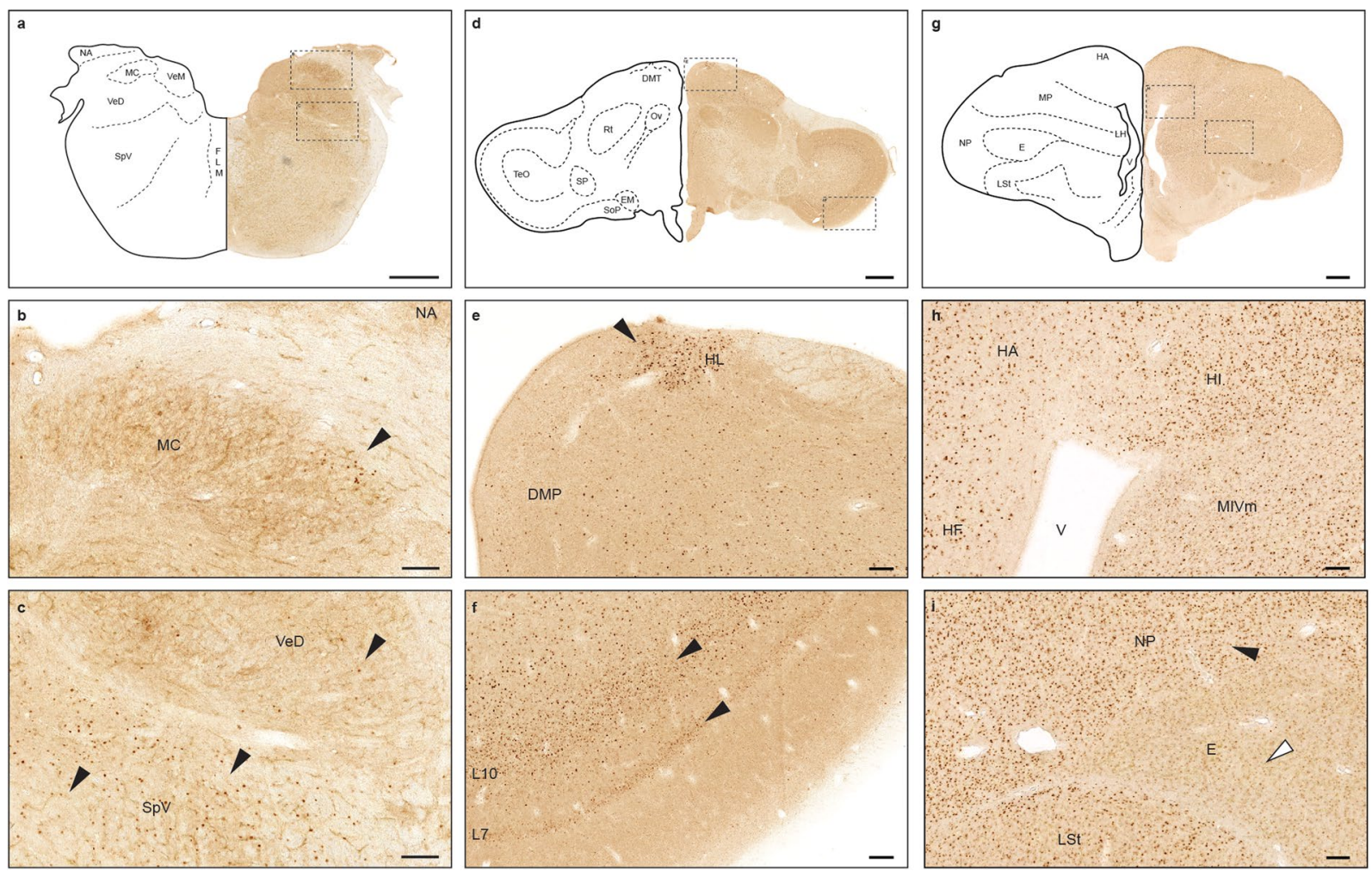

Figure 2. $\mathrm{ZENK}^{+}$neuronal populations in the pigeon brain. Pigeon brain sections at Karten/Hodos coordinates P1.25 (a), A5.00 (d), and A9.25 (g) stained with the 7B7-A3 antibody. Insets (dashed boxes) are shown below the corresponding images. In the absence of stimulation ZENK-positive nuclei can be detected throughout the pigeon brain in the (b) nucleus magnocellularis; (c) nucleus vestibularis descendens (VeD) and spinal trigeminal nucleus $(\mathrm{SpV})$; (e) lateral habenular nucleus (HL) and nucleus dorsomedialis posterior thalami (DMP); (f) layer 7 and 10 of the tectum opticum; (h) hyperpallium apicale (HA), hippocampal formation (HF), hyperpallium intercalatum (HI), and mesopallium intermedioventrale, pars medialis (MIVm); and (i) nidopallium (N) and striatum laterale (LSt). Black arrowheads highlight ZENK positive neuronal populations. The white arrowhead in (i) highlights the lack of ZENK staining in the entopallium (E). Scalebars represent $1 \mathrm{~mm}$ in upper row and $100 \mu \mathrm{m}$ in insets.

brain, including neuronal populations of the vestibular, trigeminal, and auditory, and visual system, thalamic relay stations, and telencephalic structures. This provides an important entry point for the mapping of functional activity of these networks using 7B7-A3.

The 7B7-A3 antibody is a neuronal activity marker. To assess whether the 7B7-A3 antibody can be used to detect stimulus-induced ZENK expression, we challenged restrained pigeons $(n=6)$ with a 60 min auditory stimulus consisting of pure tones, sweeps, and white noise $(2-10 \mathrm{kHz}$, Fig. 3a,b). Control animals were kept for $60 \mathrm{~min}$ in silence (control, $\mathrm{n}=6$ ). The birds were then immediately perfused, the brains sliced, and matched sections containing the cochlear nuclei (i.e. the nuclei angularis, NA, and magnocellularis, NM) were stained with the 7B7-A3 antibody. As the NA and NM represent the first stations of the central auditory pathway in the pigeon and receive direct input from cochlear afferents, we predicted that sound exposure would lead to an increase in ZENK positive cells in these brain regions. Blind quantitation revealed a 4 -fold increase in ZENK positive cells in the NA when comparing stimulated and control animals $(\mathrm{P}<0.0001 ; \mathrm{t}=8.964 ; \mathrm{df}=10$; Fig. 3c-e). Consistent with the proposed tonotopic organization within the NA in birds ${ }^{37}$, the high frequency sound-induced activation was most pronounced in the anterolateral divisions of the nucleus. Similarly, we found a significant upregulation of ZENK positive cells in the rostral parts of the NM, although ZENK expression was sparse in this area $(\mathrm{P}<0.05$; $\mathrm{t}=2.891 ; \mathrm{df}=10$; Fig. $3 \mathrm{f}-\mathrm{h}$ ). As the NA and NM project directly to the lemniscal complex in the caudal mesencephalon, which is the first station receiving binaural information, we also quantified ZENK expression in the nucleus ventralis lemnisci lateralis (VLV). Again, we found a significant increase in ZENK positive cells in stimulated animals $(\mathrm{P}<0.05 ; \mathrm{t}=2.668 ; \mathrm{df}=10$; Fig. 3i-k). We did not observe an increase in ZENK positive cells in the non-auditory trigeminal nucleus $\mathrm{SpV}$ in response to sound $(\mathrm{P}>0.05 ; \mathrm{t}=0.690 ; \mathrm{df}=10$; Fig. 31-n). Together, these results show that the 7B7-A3 antibody can be employed as a marker for stimulus-induced neuronal activity.

7B7-A3 labeling in other birds. Having established 7B7-A3 as a reliable marker for neuronal activity in the pigeon, we tested whether the antibody is compatible with staining in other avian model species. Using our standard immunohistochemical labeling procedure, we assessed the staining quality in the pigeon, zebra finch (Taeniopygia guttata), and domestic chicken (Gallus gallus domesticus). We collected brain sections from all birds 
a

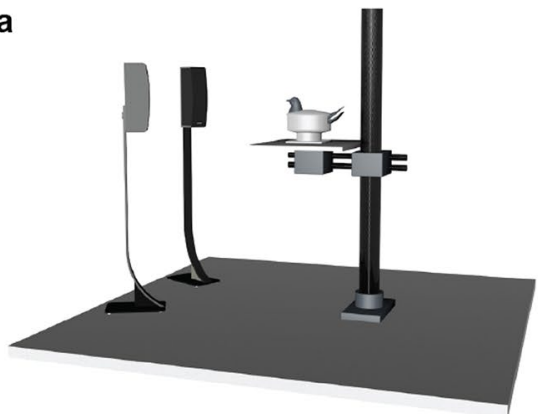

C

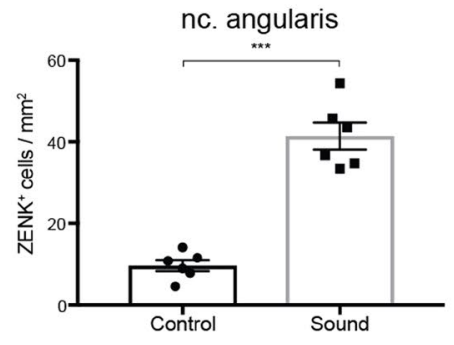

$\mathbf{f}$

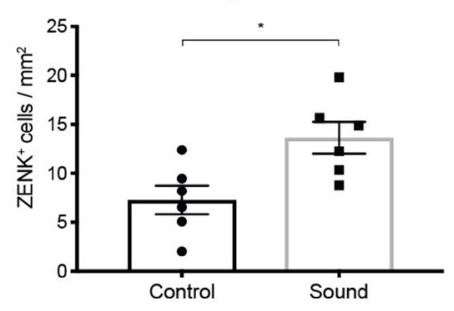

i

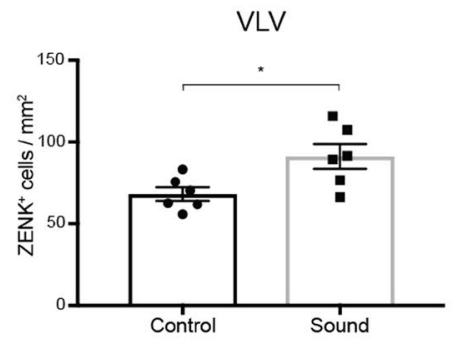

I

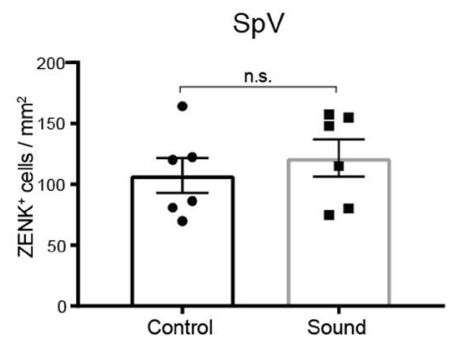

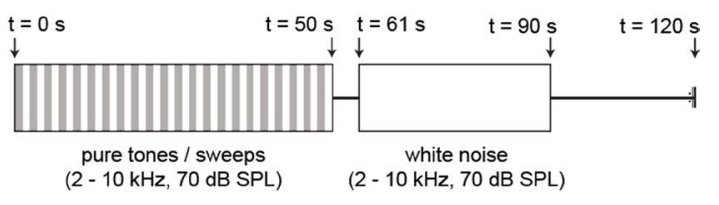

d
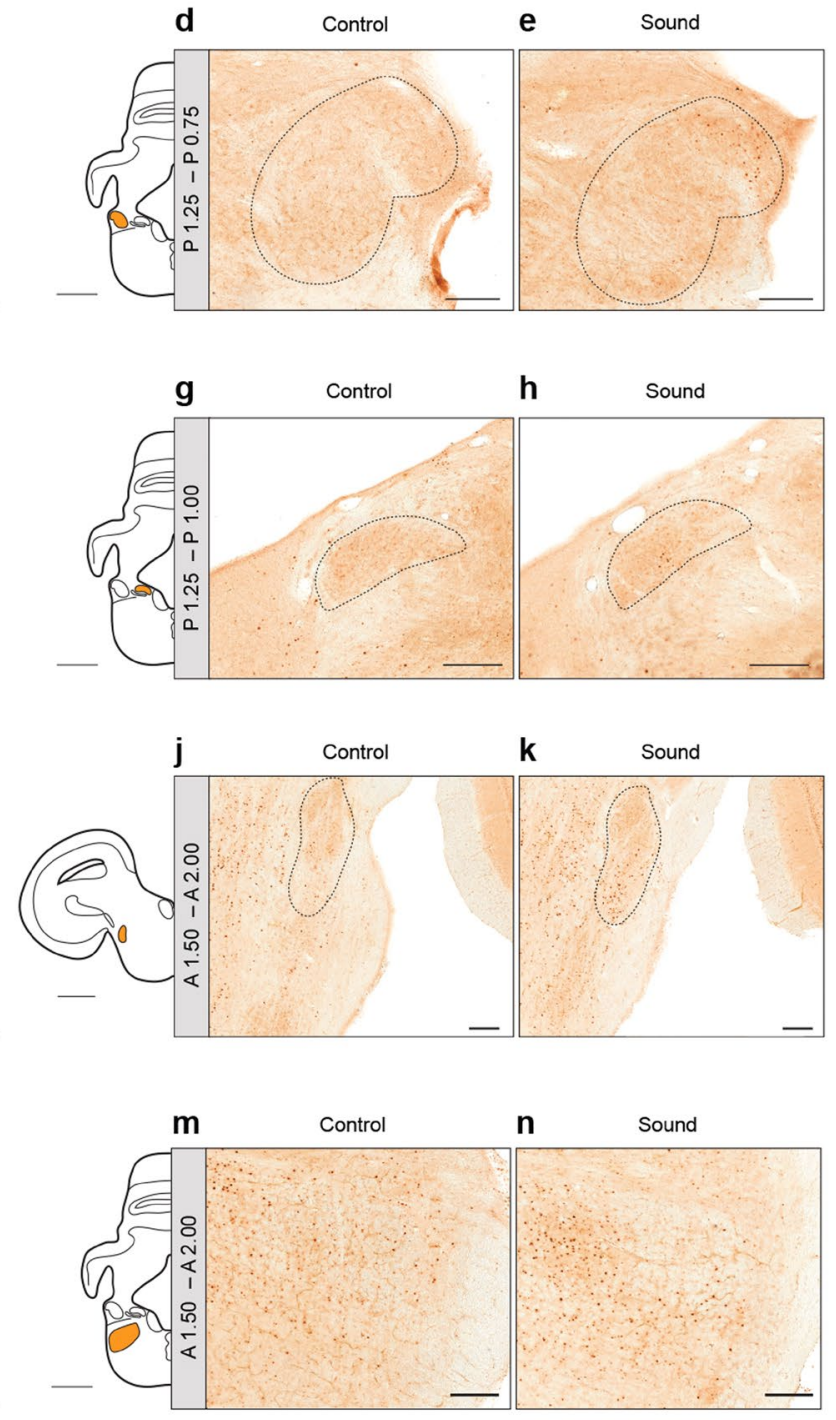

n Sound

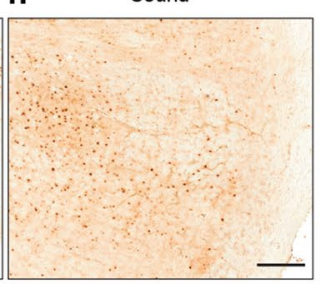

Figure 3. The 7B7-A3 antibody is a neuronal activity marker. (a) Set-up for auditory activation experiment. Pigeons were immobilized in a body harness and exposed to high frequency sounds delivered through two speakers at $1.5 \mathrm{~m}$ distance to the animals $(n=6)$ or to no sound $(n=6$, Control). (b) Sound stimulation paradigm: T1-t50: double-pulsed pure tones and sweeps (each $50 \mathrm{~ms}, 2-10 \mathrm{kHz}, 70 \mathrm{~dB} \mathrm{SPL}$ ), delivered in a pseudorandomized order; t51-t60: silence; t61-t90: white noise (2-10 kHz, $70 \mathrm{~dB}$ SPL); t91-t120: silence. The sound file was looped 30 times to achieve a $60 \mathrm{~min}$ stimulation. (c-e) Quantification of ZENK ${ }^{+}$cells per $\mathrm{mm}^{2}$ in the nucleus angularis, (f-h) nucleus magnocellularis, (i-k) nucleus ventralis lemnisci lateralis (VLV), and (l-n) spinal trigeminal nucleus (SpV). Right panels show representative coronal sections from control and stimulated animals stained with the 7B7-A3 antibody as well as the segmented regions for the nucleus angularis $(\mathbf{d}, \mathbf{e})$, nucleus magnocellularis $(\mathbf{g}, \mathbf{h}), \operatorname{VLV}(\mathbf{j}, \mathbf{k})$, and $\operatorname{SpV}(\mathbf{m}, \mathbf{n})$. On the left of $(\mathbf{d}),(\mathbf{g}),(\mathbf{j})$, and (m), anatomical drawings of coronal hemisections with the segmented nuclei are shown in orange and stereotaxic coordinates according to Hodos and Karten ${ }^{50}$ are indicated in the grey boxes. Data are presented as mean $+/-$ SD. $* \mathrm{P}<0.05 ; * * * \mathrm{P}<0.0001$, one tailed t-test after Bonferroni-correction in auditory areas. n.s.: not significant. Scalebars represent $2 \mathrm{~mm}$ in the drawings and $200 \mu \mathrm{m}$ in stained sections. 
$(n=3)$ and focused on the distribution of ZENK positive cells in the caudal hippocampus and surrounding structures. In pigeons we observed ZENK positive nuclei in all divisions of the caudal hippocampus as well as in the parahippocampal area (Fig. 4b,c). Using 7B7-A3 at the same concentration in the zebra finch $(1: 500,000)$ we again observed nuclear staining. Compared to pigeons this staining was sparser in the hippocampus, but more pronounced in the ventrally located mesopallium (Fig. 4e) and the parahippocampal area (Fig. 4e,f). In the chicken, a higher concentration of the antibody (1:5000) was required to achieve immunolabelling in the hippocampus (Fig. 4h). There, ZENK positive cells were less intensely stained, but again showed nuclear signal. Similarly to the zebra finch, the expression in mesopallial regions was more pronounced (Fig. 4i). While context dependent ZENK expression could account for the observed differences in ZENK distribution between the species, these results confirm that 7B7-A3 is compatible with immunohistochemical detection of ZENK in the rock pigeon, zebra finch, and chicken, thereby broadening the utility of the 7B7-A3 antibody.

\title{
Discussion
}

Here, we present a new monoclonal antibody (7B7-A3) that targets the ZENK protein from the rock pigeon. This antibody has several salient features. First, it is the only antibody that has been specifically raised against a bird ZENK protein. The antibody is compatible with western blot analysis and immunohistochemistry and shows a robust signal to noise ratio under denaturating conditions. Importantly, selective binding of the antibody to the antigen (as recommended by the international working group for antibody validation ${ }^{38}$ ) demonstrates that it binds to ZENK. Second, the 7B7-A3 antibody exhibits high sensitivity for its target, as evident from the immunohistochemical results. On pigeon brain tissue sections, a dilution of 1:500,000 is sufficient to label neuronal nuclei without intensification by heavy metals. This high sensitivity ensures thorough and quantifiable labeling of activated brain areas, but also the identification of neuronal populations in which ZENK expression has not been described before, such as the nuclei angularis and magnocellularis. Third, the wide distribution of ZENK positive cells in the pigeon brainstem detected by the 7B7-A3 antibody make it an ideal tool to study the functional organization of many sensory entry stations, such as the trigeminal, auditory, or vestibular systems. For instance, the anatomical localization of sound-induced activity in the pigeon has so far been focused on telencephalic structures such as Field $\mathrm{L}^{39}$ or the nucleus basalis ${ }^{40}$ and in the midbrain on the nucleus mesencephalic lateralis pars dorsalis ${ }^{41}$. Challenging the assertions that IEG induction does not occur in the cochlear nuclei of birds ${ }^{12}$, we have shown the 7B7-A3 antibody does label the pigeon auditory brainstem in an activity-based manner. Fourth, 7B7-A3 recognizes ZENK epitopes from different bird species, broadening its utility to numerous research fields associated with the avian brain. Studies of the zebra finch song system have greatly benefitted from ZENK mapping $^{8,12}$, and in the pigeon, ZENK induced by homing behavior has been used to study the neuronal correlates of navigational processes ${ }^{29,42}$. Finally, with the advent of brain clearing methods, such as CUBIC ${ }^{43}$, CLARITY $^{44}$, or iDISCO $^{45}$, the 7B7-A3 antibody may also serve as a useful tool to investigate IEG expression on a global scale ${ }^{46,47}$. Such experiments will provide further insight into the neuronal circuits that mediate the complex repertoire of avian behaviours. Previous studies that have employed ZENK as a surrogate marker for neuronal activity have primarily relied on a polyclonal antibody (C-19), which has now been discontinued by the supplier ${ }^{27,28}$. We therefore anticipate that the 7B7-A3 ZENK monoclonal antibody described in this manuscript will be a valuable tool for the avian neuroscience community. This antibody is available on request.

\begin{abstract}
Methods
Identification and amplification of the clZENK sequence. Brain tissue samples were collected from adult rock pigeons (Columba livia), snap frozen in liquid nitrogen, and mechanically homogenized in a Tissue Lyser (Qiagen, 85300). Following the manufacturers' instructions, total RNA was extracted from the lysates using the RNeasy mini kit (Qiagen, 74104) and reversely transcribed to cDNA using the Superscript III cDNA synthesis SuperMix kit (Invitrogen, 18080400). The resulting pigeon brain cDNA library was diluted to a working concentration of $1: 100$ and stored at $-20^{\circ} \mathrm{C}$. Sets of overlapping polymerase chain reaction (PCR) primers for the predicted $c l Z E N K$ transcripts were designed based on available genomic resources. PCR amplification of the pigeon brain cDNA library was performed in two segments with the Phusion Hot Start Flex DNA polymerase (NEB, M0535S) using the following primer sequences: segment 1: Fwd: ATGGACACTCACTATCCCAA, Rev: GCAAACGCCTTAATAGTGG; segment 2 Fwd: CCACTATTAAGGCGTTTGC, Rev: GGACAAC TGAGATTTGCTGA. PCR products were evaluated by agarose gel electrophoresis, TA-cloned into the pCR4-TOPO vector (Invitrogen, K457502) following the manufacturer's protocol, and analyzed by Sanger sequencing. For protein expression, a C-terminal enhanced green fluorescence protein (eGFP) tag was fused to the total clZENK nucleotide sequence after a four residue glycine-serine linker and cloned into the mammalian expression vector pCIneo. Gibson assembly was performed according to the manufacturer's (MBS VBC) protocol with the following fragments: pCIneo plasmid linearized with NheI/XhoI restriction enzymes (NEB, R0131S and R0146S); clZENK amplified in two fragments using Phusion Hot Start Flex DNA polymerase; eGFP amplified from a host plasmid using Phusion Hot Start Flex DNA polymerase. The full coding sequence of the construct was verified by Sanger sequencing.
\end{abstract}

Monoclonal antibody generation. For the generation of a monoclonal antibody against $c l Z E N K$, a 260 amino acid protein fragment (1-260) was selected as antigen; the codon-optimized mRNA was synthesized and cloned into the pET23a(+) expression vector including a C-terminal hexahistidine tag by an external contractor (GenScript USA Inc). The peptide was recombinantly expressed in Lemo21(DE3) bacteria (NEB, C2528J) and purified from the insoluble fraction of the bacterial lysates in $8 \mathrm{M}$ urea over a Nickel-sepharose column (GE Healthcare, 17526801) and the Whatmam ${ }^{\mathrm{TM}}$ Elutrap $^{\mathrm{TM}}$ electroelution system (Thermo Fischer, 15560753). The eluate was verified by mass spectrometry. 
a

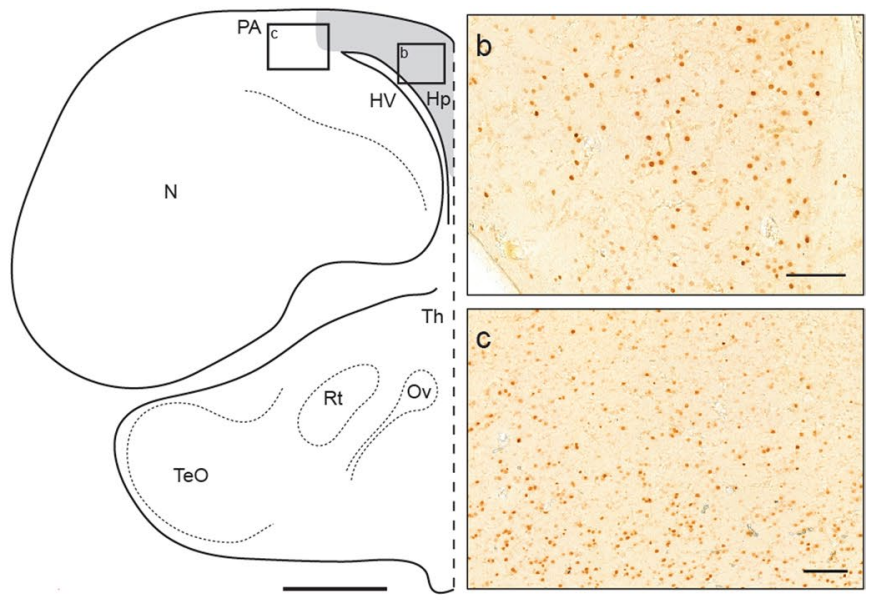

d

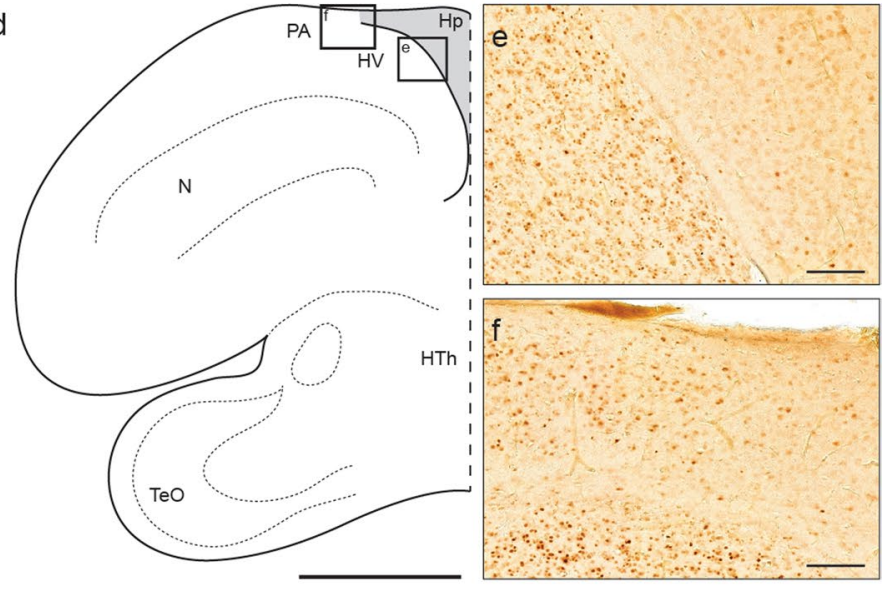

g

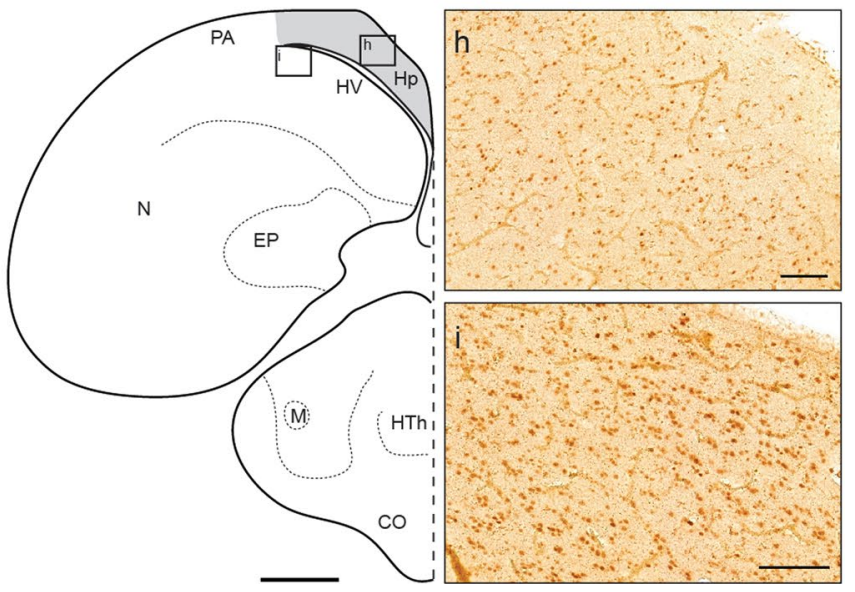

Figure 4. Hippocampal 7B7-A3 labeling in different avian species. Schematic representations of a coronal brain section of the (a) rock pigeon, (d) zebra finch, and (g) domestic chicken. The hippocampus is highlighted in grey; insets are shown on the right. Staining with 7B7-A3 antibody in the pigeon leads to robust nuclear staining within the entire (b) hippocampus and (c) parahippocampal area. In the zebra finch, hippocampal neurons show only sparse ZENK staining (e), while robust nuclear signal can be detected in the mesopallium as well as in the (f) parahippocampal area. In the chicken, 7B7-A3 stains the (h) hippocampus uniformly, but less intensely than those cells in the (i) parahippocampal area. Scalebars represent $2 \mathrm{~mm}$ in (a), (d), and (g); and 100 $\mu \mathrm{m}$ in $(\mathbf{b}),(\mathbf{c}),(\mathbf{e}),(\mathbf{f}),(\mathbf{h})$, and (i). 
$\mathrm{BALB} / \mathrm{c}$ mice were immunized with the purified antigen. Following the hybridoma technique ${ }^{48}$, monoclonal antibody producing cells were generated and positive clones were isolated after several rounds of monoclonal selection by testing cell culture supernatants for immune reactivity in western blots and on tissue sections (see below). Hybridoma cells of the final clone, 7B7-A3, were weaned from HAT (Gibco, 21060017) in a stepwise process over 2 weeks. For antibody expression, a hollow fiber cartridge (Fiber Cell Systems, C2011) was inoculated according to manufacturer's instructions and the cells were cultivated using production medium containing DMEM (Media kitchen, VBCF) supplemented with 5\% FCS (Gibco, 16140071), 1\% Penicillin-Streptomycin (Gibco, 15140122), 2 mM L-Glutamine (Gibco, 25030081), and $1 \mathrm{mM}$ sodium pyruvate (Gibco, 11360070). Supernatants were harvested and pooled for 5 weeks; the monoclonal antibody was isolated by affinity purification using a Protein G column (elution buffer: $100 \mathrm{mM}$ Glycine, $100 \mathrm{mM} \mathrm{NaCl}, \mathrm{pH} 2.46$ ).

Cell culture, protein expression and western blot analysis. Primary cell cultures of pigeon embryonic fibroblasts (PEF) isolated at embryonic day 10 were cultured at $37^{\circ} \mathrm{C}$ and $5 \% \mathrm{CO}_{2}$ on $10 \mathrm{~cm}$ cell culture dishes (Eppendorf) in DMEM (MBS VBC) supplemented with 8\% chicken serum (C5405), 2\% FCS (Gibco, 16140071), $1 \%$ penicillin-streptomycin (Gibco, 15140122), 1\% L-Glutamine (Gibco, 25030081), 1\% non-essential amino acids (Gibco, 11140050), sodium pyruvate (Gibco, 11360070), and $70 \mathrm{mM} \beta$-mercaptoethanol (Merck, 805740). Cells were transfected at $\sim 80 \%$ confluence with $20 \mu \mathrm{g}$ pCIneo-ZENK-eGFP or a control construct (pCIneo-GFP) using Lipofectamine 2000 transfection reagent (Invitrogen, 11668019) according to the manufacturer's instructions. $24-48 \mathrm{~h}$ post transfection, cells were washed twice in PBS and harvested in $250 \mu \mathrm{l}$ RIPA buffer containing $150 \mathrm{mM} \mathrm{NaCl}, 1 \%$ Triton X-100 (Sigma Aldrich, X100), 0.5\% sodium deoxycholate (Sigma Aldrich, D6750), 0.1\% SDS (Sigma Aldrich, 862010), $50 \mathrm{mM}$ Tris $\mathrm{pH}$ 8, and one protease inhibitor tablet (Pierce, A2963). Cells were snap frozen in liquid nitrogen, thawed, lysed for one hour shaking at $4{ }^{\circ} \mathrm{C}$, and centrifuged for $30 \mathrm{~min}$ at $4{ }^{\circ} \mathrm{C}$ $(16200 \mathrm{~g})$. Supernatants were collected and total protein concentrations were determined using the BCA Protein Assay kit (Pierce, 23225). For western blot analysis, $20 \mu \mathrm{g}$ of protein extracts were denatured in Laemmli sample buffer (BIO-RAD, 1610747) for $5 \mathrm{~min}$ at $95^{\circ} \mathrm{C}$ and separated in a $4-12 \%$ bis-tris poly-acrylamide gel (Invitrogen. NP0322). Gel electrophoresis was performed for $2 \mathrm{~h}$ at $120 \mathrm{~V}$ in MOPS SDS running buffer (Invitrogen, NP0001) and subsequent transferred to a PVDF membrane (Merck, ISEQ. 00010) for $3 \mathrm{~h}$ at $100 \mathrm{~V}$ and $4{ }^{\circ} \mathrm{C}$ in transfer buffer (30 mM tris, $240 \mathrm{mM}$ glycine, $0.025 \%$ SDS). After rinsing in Tris-buffered saline supplemented with $0.1 \%$ Tween20 (Sigma Aldrich, P1379) (TBST) and blocking for $1 \mathrm{~h}$ in TBST supplemented with 5\% non-fat milk powder, membranes were incubated in primary antibody solution composed of $5 \%$ milk/TBST over night at $4{ }^{\circ} \mathrm{C}$. The following concentrations were used: 1:500 (mouse serum screen), 1:2000 (7B7-A3 supernatant), 1:500 GFP (2B6, Monoclonal Antibody Facility, Max Perutz Laboratories). After washes in TBST, membranes were incubated for $1 \mathrm{~h}$ with the respective HRP-tagged secondary antibody (Abcam, ab6823) in 5\% milk/TBST, washed, and imaged using the ECL western blotting detection reagents (Amersham, RPN2232) on a BIORAD ChemiDOC ${ }^{\mathrm{TM}}$ imager. For the antigen competition experiment, $4 \mu \mathrm{g}$ of the final ZENK antibody was incubated with $20 \mu \mathrm{g}$ of the immunogen for $2 \mathrm{~h}$, before the antibody-antigen solution was used as described above in primary antibody solution.

Avian tissue collection for immunohistochemistry. Adult pigeons (Columba livia) were maintained on a 12:12 light-dark cycle at $25^{\circ} \mathrm{C}$ in a custom-built aviary. Zebra finches (Taeniopygia guttata) and domestic chicken (Gallus gallus domesticus) were purchased from local suppliers and kept in individual transport boxes for at least 1 hour prior to sacrifice. Animals used to assess ZENK expression in the absence of sensory stimulation were individually housed for at least 7 hours in total darkness and silence. For brain tissue collection birds were sacrificed and transcardially perfused with $200 \mathrm{ml}$ sterile filtered, warm PBS supplemented with $15 \mathrm{kU}$ Heparin (Carl Roth, 7692.2) followed by $200 \mathrm{ml}$ of $4 \%$ PFA in phosphate buffer (Sigma Aldrich, 158127). The brains were dissected, postfixed in $4 \%$ PFA for 18 hours at $4^{\circ} \mathrm{C}$, dehydrated in $30 \%$ sucrose in $0.1 \mathrm{M} \mathrm{PBS}$ (Sigma Aldrich, S9378) for 3 days at $4{ }^{\circ} \mathrm{C}$, and mounted on a sledge microtome. $40 \mu \mathrm{m}$ serial sections were acquired in the coronal plane and stored in anti-freeze composed of PBS supplemented with 30\% Glycerol (AppliChem, A0970) and 30\% Ethyleneglycol (Sigma Aldrich, 324558) at $-20^{\circ} \mathrm{C}$. For broad assessment of basal ZENK expression in the pigeon, at least 16 tissue sections were mounted on glass slides (Thermo Fischer Scientific, J4800AMNZ). For the comparison of ZENK expression in the pigeon, zebra finch, and chicken, individual hippocampal sections of each species were mounted on the same slide. For all experiments at least three animals were used. All sections were dried over night before proceeding with immunohistochemical staining.

Ethical framework. All experiments were carried out in accordance with relevant guidelines and regulations. Experimental protocols were approved by an existing ethical framework (GZ: 214635/2015/20) granted by the City of Vienna (Magistratsabteilung 58).

Auditory activation. Sound activation experiments were performed in an experimental chamber in darkness. Birds were habituated to a customised body harness and experimental room for 30 minutes on 3 consecutive days prior to the experiment. The night prior to the experiment birds were singularly housed. Single animals were placed in the center of the chamber in the body harness on an elevated platform, with 2 speakers (Sony, SS-MD333) placed $1.5 \mathrm{~m}$ away from the birds. Two birds were tested on each day and were presented with either a $60 \mathrm{~min}$ sound file or no sound. The experimenter was blind to the stimulation condition at all times. The sound stimulus was generated using the open source software Audacity (D. Mazzoni) and consisted of a $2 \mathrm{~min}$ high activity sequence that was looped 30 times to achieve a 60 min stimulation ${ }^{49}$. The sequence was composed of: (start to $t=50 \mathrm{~s}$ ): double-pulsed sine waves (each $50 \mathrm{~ms}, 2-10 \mathrm{kHz}, 70 \mathrm{~dB} \mathrm{SPL}$ ) and sweeps (each $50 \mathrm{~ms}$, $2-10 \mathrm{kHz}, 70 \mathrm{~dB}$ SPL) delivered in a pseudorandomized order; $(t=51 \mathrm{~s}$ to $t=60 \mathrm{~s})$ : silence; $(t=61 \mathrm{~s}$ to $t=90 \mathrm{~s})$ : white noise $(2-10 \mathrm{kHz}, 70 \mathrm{~dB} \mathrm{SPL}) ;(t=91 \mathrm{~s}$ to $t=120 \mathrm{~s})$ : silence. A habituation period prior to stimulation to exclude handling-induced ZENK expression was omitted due to the strong activation profile of the sound stimulus. Following stimulus presentation birds were immediately sacrificed and perfused and brains were processed 
as described above. For the analysis of sound-induced ZENK expression in the auditory brainstem, every third section of the cochlear nuclei was mounted on glass slides starting the at the caudal border of the nucleus magnocellularis $^{50}$ (P 1.50). In addition, five sections of the isthmic brainstem containing VLV were mounted starting at A 1.50. All sections were dried overnight before proceeding with immunohistochemical staining.

Immunohistochemistry. Mounted and dried sections were washed $3 \times 5 \mathrm{~min}$ in PBS before heat-based antigen retrieval was performed to unmask fixed antigens following the manufacturer's instructions (Vector Laboratories, H-3301). After a $30 \mathrm{~min}$ cooling period, slides were washed $3 \times 5 \mathrm{~min}$ in PBS and incubated in primary antibody diluted in $0.3 \%$ Triton-X100/PBS with $4 \%$ milk powder over night at room temperature. The following concentrations were used: 1:5 (mix clone supernatants), 1:100 (final clone supernatant), 1:500,000 (7B7-A3, pigeon), 1:500,000 (7B7-A3, zebra finch), 1:5000 (7B7-A3, chicken). The next day, slides were washed $3 \times 5$ minutes in PBS, incubated with the secondary antibody (1:1000, anti-mouse, Vector Laboratories, PK-6100) for 2 hours at room temperature, washed, and incubated with the Vectastain Elite ABC HRP reagent (Vector Laboratories, PK-6100) for $1 \mathrm{~h}$. After another round of washing, slides were incubated for $1 \mathrm{~min}$ in PBS supplemented with $0.06 \%$ Diaminobenzidine (Sigma Aldrich, D5905) and $0.08 \% \mathrm{H}_{2} \mathrm{O}_{2}$. After a final washing step, slides were dehydrated in serial dilutions of ethanol and cover slipped.

Counting and statistical analysis. Slides were scanned on a slide scanner with a 20x objective (Pannoramic 250 Flash III, 3DHistech). All auditory nuclei were identified based on surrounding white matter tracts and segmented manually (at least 8 bilateral segments from 4 sections) using Pannoramic Viewer (Pannoramic Viewer 1.15.4, 3DHistech). ZENK expression in SpV was assessed on 3 sections (6 bilateral segments). Automated identification and counting of ZENK positive nuclei on exported segments were performed by custom made rule sets using a machine-learning algorithm embedded in the Definiens Architect software (Definiens Architect XD, Definiens Software). The number of ZENK positive cells per $\mathrm{mm}^{2}$ was calculated based on the area of exported segments. Context dependent changes in ZENK expression were assessed using a one-sided t-test and sound-induced changes in ZENK expression were assessed using one-sided t-tests corrected for multiple comparisons (Bonferroni) in auditory areas. Statistical analyses were performed in R (Team 2013) and figures were generated using Graphpad Prism 8 (Graphpad Software Inc).

\section{Data availability}

The datasets generated during and/or analysed during the current study are available from the corresponding author on reasonable request.

Received: 7 August 2019; Accepted: 20 December 2019;

Published online: 22 January 2020

\section{References}

1. Reiner, A. et al. The Avian Brain Nomenclature Forum: Terminology for a New Century in Comparative Neuroanatomy. J. Comp. Neurol. 473, E1-E6 (2004).

2. Weir, A. A., Chappell, J. \& Kacelnik, A. Shaping of hooks in New Caledonian crows. Science 297, 981 (2002).

3. Emery, N. J. \& Clayton, N. S. The mentality of crows: convergent evolution of intelligence in corvids and apes. Science 306, 1903-7 (2004).

4. Auersperg, A. M., Kacelnik, A. \& von Bayern, A. M. Explorative learning and functional inferences on a five-step means-means-end problem in Goffin's cockatoos (Cacatuagoffini). PLoS One 8, e68979 (2013).

5. Vonfersen, L. \& Delius, J. D. Long-Term Retention of Many Visual-Patterns by Pigeons. Ethology 82, 141-155 (1989).

6. Güntürkün, O., Stüttgen, M. C. \& Manns, M. Pigeons as a model species for cognitive neuroscience. e-Neuroforum 5, 86-92 (2014).

7. Chew, S. J., Vicario, D. S. \& Nottebohm, F. A large-capacity memory system that recognizes the calls and songs of individual birds. Proc. Natl Acad. Sci. USA 93, 1950-5 (1996).

8. Bolhuis, J. J. \& Gahr, M. Neural mechanisms of birdsong memory. Nat. Rev. Neurosci. 7, 347-57 (2006).

9. Wiltschko, W. \& Wiltschko, R. Magnetic compass of European robins. Science 176, 62-4 (1972).

10. Mouritsen, H., Heyers, D. \& Gunturkun, O. The Neural Basis of Long-Distance Navigation in Birds. Annu. Rev. Physiol. 78, 133-54 (2016).

11. Kaczmarek, L. \& Chaudhuri, A. Sensory regulation of immediate-early gene expression in mammalian visual cortex: implications for functional mapping and neural plasticity. Brain Res. Brain Res Rev. 23, 237-56 (1997).

12. Mello, C.V. \& Pinaud, R. In Immediate early genes in sensory processing, cognitive performance and neurological disorders 35-56 (Springer, 2006).

13. Milbrandt, J. A nerve growth factor-induced gene encodes a possible transcriptional regulatory factor. Science 238, 797-9 (1987).

14. Christy, B. A., Lau, L. F. \& Nathans, D. A gene activated in mouse 3 T3 cells by serum growth factors encodes a protein with "zinc finger" sequences. Proc. Natl Acad. Sci. USA 85, 7857-61 (1988).

15. Lemaire, P., Revelant, O., Bravo, R. \& Charnay, P. Two mouse genes encoding potential transcription factors with identical DNAbinding domains are activated by growth factors in cultured cells. Proc. Natl Acad. Sci. USA 85, 4691-5 (1988).

16. Sukhatme, V. P. et al. A zinc finger-encoding gene coregulated with c-fos during growth and differentiation, and after cellular depolarization. Cell 53, 37-43 (1988).

17. Velho, T. A. \& Mello, C. V. Synapsins are late activity-induced genes regulated by birdsong. J. Neurosci. 28, 11871-82 (2008).

18. Mello, C. V., Vicario, D. S. \& Clayton, D. F. Song presentation induces gene expression in the songbird forebrain. Proc. Natl Acad. Sci. USA 89, 6818-22 (1992).

19. Mello, C. V. \& Clayton, D. F. Song-induced ZENK gene expression in auditory pathways of songbird brain and its relation to the song control system. J. Neurosci. 14, 6652-66 (1994).

20. Mello, C. V. \& Ribeiro, S. ZENK protein regulation by song in the brain of songbirds. J. Comp. Neurol. 393, 426-438 (1998).

21. Mello, C. V. \& Clayton, D. F. Differential induction of the ZENK gene in the avian forebrain and song control circuit after metrazoleinduced depolarization. J. Neurobiol. 26, 145-161 (1995).

22. Feenders, G. et al. Molecular mapping of movement-associated areas in the avian brain: a motor theory for vocal learning origin. PLoS one 3, e1768 (2008).

23. Mello, C. V. \& Jarvis, E. D. In Neuroscience of Birdsong. (eds. Zeigler, H. P. \& Marler, P.) (Cambridge University Press, Cambridge, 2008).

24. Kruse, A. A., Stripling, R. \& Clayton, D. F. Context-specific habituation of the zenk gene response to song in adult zebra finches. Neurobiol. Learn. Mem. 82, 99-108 (2004). 
25. Avey, M. T., Phillmore, L. S. \& MacDougall-Shackleton, S. A. Immediate early gene expression following exposure to acoustic and visual components of courtship in zebra finches. Behavioural brain Res. 165, 247-253 (2005).

26. Velho, T. A. et al. Noradrenergic control of gene expression and long-term neuronal adaptation evoked by learned vocalizations in songbirds. PLoS one 7, e36276 (2012).

27. Heyers, D., Zapka, M., Hoffmeister, M., Wild, J. M. \& Mouritsen, H. Magnetic field changes activate the trigeminal brainstem complex in a migratory bird. Proc. Natl Acad. Sci. USA 107, 9394-9 (2010).

28. Lefeldt, N. et al. Magnetic field-driven induction of ZENK in the trigeminal system of pigeons (Columba livia). J. R. Soc. Interface 11, 20140777 (2014).

29. Patzke, N., Manns, M., Güntürkün, O., Ioale, P. \& Gagliardo, A. Navigation-induced ZENK expression in the olfactory system of pigeons (Columba livia). Eur. J. Neurosci. 31, 2062-2072 (2010).

30. Heyers, D., Manns, M., Luksch, H., Güntürkün, O. \& Mouritsen, H. A visual pathway links brain structures active during magnetic compass orientation in migratory birds. PLoS One 2, e937 (2007).

31. Holt, C. et al. Improved Genome Assembly and Annotation for the Rock Pigeon (Columba livia). G3 (Bethesda) 8, 1391-1398 (2018).

32. Shapiro, M. D. et al. Genomic diversity and evolution of the head crest in the rock pigeon. Science 339, 1063-7 (2013).

33. Patzke, N., Manns, M. \& Güntürkün, O. Telencephalic organization of the olfactory system in homing pigeons (Columba livia). Neuroscience 194, 53-61 (2011).

34. Atoji, Y., Sarkar, S. \& Wild, J. M. Differential projections of the densocellular and intermediate parts of the hyperpallium in the pigeon (Columba livia). J. Comp. Neurol. 526, 146-165 (2018).

35. Aoki, N. et al. Critical role of the neural pathway from the intermediate medial mesopallium to the intermediate hyperpallium apicale in filial imprinting of domestic chicks (Gallus gallus domesticus). Neuroscience 308, 115-124 (2015).

36. Herold, C. et al. Distribution of neurotransmitter receptors and zinc in the pigeon (Columba livia) hippocampal formation: A basis for further comparison with the mammalian hippocampus. J. Comp. Neurol. 522, 2553-2575 (2014).

37. Köppl, C. Tonotopic projections of the auditory nerve to the cochlear nucleus angularis in the barn owl. JARO-Journal Assoc. Res. Otolaryngology 2, 41-53 (2001).

38. Uhlen, M. et al. A proposal for validation of antibodies. Nat. Methods 13, 823-7 (2016).

39. Muller, S. C. \& Scheich, H. Functional-Organization of the Avian Auditory Field-L - a Comparative 2dg Study. J. Comp. Physiol. a-Sensory Neural Behav. Physiol. 156, 1-12 (1985).

40. Maekawa, M. Auditory responses in the nucleus basalis of the pigeon. Hearing Res. 27, 231-237 (1987)

41. Lewald, J. Neural mechanisms of directional hearing in the pigeon. Exp. brain Res. 82, 423-436 (1990).

42. Shimizu, T., Bowers, A. N., Budzynski, C. A., Kahn, M. C. \& Bingman, V. P. What does a pigeon (Columba livia) brain look like during homing? selective examination of ZENK expression. Behav. Neurosci. 118, 845 (2004).

43. Susaki, E. A. et al. Whole-brain imaging with single-cell resolution using chemical cocktails and computational analysis. Cell 157, 726-739 (2014).

44. Chung, K. et al. Structural and molecular interrogation of intact biological systems. Nature 497, 332 (2013).

45. Renier, N. et al. iDISCO: a simple, rapid method to immunolabel large tissue samples for volume imaging. Cell 159, 896-910 (2014).

46. Renier, N. et al. Mapping of brain activity by automated volume analysis of immediate early genes. Cell 165, 1789-1802 (2016).

47. Randlett, O. et al. Whole-brain activity mapping onto a zebrafish brain atlas. Nat. methods 12, 1039 (2015).

48. Kohler, G. \& Milstein, C. Continuous cultures of fused cells secreting antibody of predefined specificity. Nature 256, 495-7 (1975).

49. Heffner, H. E., Koay, G., Hill, E. M. \& Heffner, R. S. Conditioned suppression/avoidance as a procedure for testing hearing in birds: The domestic pigeon (Columba livia). Behav. Res. methods 45, 383-392 (2013).

50. Karten, H. J. \& Hodos, W. Stereotaxic atlas of the brain of the pigeon Columba livia. (1967).

\section{Acknowledgements}

DAK is supported by the European Research Council (ERC, 336725) and the FWF (Y726). SN is a recipient of a DOC Fellowship of the Austrian Academy of Sciences at the Research Institute of Molecular Pathology. We wish to thank Boehringer Ingelheim who fund basic scientific research at the Research Institute of Molecular Pathology. We would like to thank the excellent support facilities at the IMP and VBCF including: molecular biology service, mass spectroscopy facility, histology, and bio-optics.

\section{Author contributions}

G.C.N. and D.A.K. designed the study, G.C.N., S.N., E.P.M. and L.U. performed the experiments, R.H., S.S., E.O. generated the ZENK antibody., L.L. undertook the statistical analysis, G.C.N. prepared the figures, G.C.N. and D.A.K. wrote the manuscript, all authors reviewed the manuscript.

\section{Competing interests}

The authors declare no competing interests.

\section{Additional information}

Supplementary information is available for this paper at https://doi.org/10.1038/s41598-020-57757-6.

Correspondence and requests for materials should be addressed to D.A.K.

Reprints and permissions information is available at www.nature.com/reprints.

Publisher's note Springer Nature remains neutral with regard to jurisdictional claims in published maps and institutional affiliations.

Open Access This article is licensed under a Creative Commons Attribution 4.0 International

License, which permits use, sharing, adaptation, distribution and reproduction in any medium or format, as long as you give appropriate credit to the original author(s) and the source, provide a link to the Creative Commons license, and indicate if changes were made. The images or other third party material in this article are included in the article's Creative Commons license, unless indicated otherwise in a credit line to the material. If material is not included in the article's Creative Commons license and your intended use is not permitted by statutory regulation or exceeds the permitted use, you will need to obtain permission directly from the copyright holder. To view a copy of this license, visit http://creativecommons.org/licenses/by/4.0/.

(C) The Author(s) 2020 